\title{
Diagnosis and Treatment of Pregnancy Concurrent with Asymptomatic Aortic Dissection
}

\author{
Shao Wenchong, Tian Ailing, Fan Lufeng, Wu Han, Sun Nianfeng \\ Department of General Surgery, Qilu Hospital, Cheeloo College of Medicine, Shandong University, Ji'nan, China
}

\section{ABSTRACT}

Background: Aortic dissection (AD) refers to false lumen dissected from true lumen via the internal membrane when endovascular blood flows into the aortic wall through the cleavage, which is formed after endomembrane gape of the aortic wall. Aortic dissection is a disease of extreme danger. Pregnancy is an independent risk factor for aortic dissection. Pregnancy concurrent with aortic dissection rarely is seen, not to mention pregnancy concurrent with asymptomatic aortic dissection.

Case report: We present the case of a 34-year-old female patient, who was 25+ weeks pregnant, concurrent with aortic dissection. She was accepted by our hospital and successfully treated. Retrospective analysis of clinical data was carried out in this paper by combining related literature.

Conclusion: This disease should be correctly straightened out to prevent misdiagnosis and save the patient's life. Multidisciplinary joint decisions should be made to save lives of the patient and her fetus.

\section{INTRODUCTION}

Aortic dissection (AD) refers to false lumen dissected from true lumen through the internal membrane when endovascular blood flows into the aortic wall through the cleavage, which is formed after endomembrane gape of the aortic wall. Aortic dissection is a disease of extreme danger. The disease attacking females is closely related to pregnancy [Katz 1984], and pregnancy itself is an independent risk factor for aortic dissection [Braverman 2010]. Pregnancy concurrent with aortic dissection rarely is seen, not to mention pregnancy concurrent with asymptomatic aortic dissection. One pregnant patient concurrent with aortic dissection recently was accepted by our hospital, successfully treated, and cured. Retrospective analysis of this clinical data was carried out in this paper by combining related literature and aimed at discussing the diagnosis and treatment of pregnancy concurrent with asymptomatic aortic dissection.

Received December 30, 2021; accepted April 19, 2021.

Correspondence: Sun Nianfeng, Department of General Surgery, Qilu Hospital of Shandong University, Fi'nan 250012, China; 086-18561810688; fax: 0860531-85695754 (e-mail: sunnianfeng@126.com).

\section{CASE REPORT}

A female patient (34 years old, 25+4-week pregnant) was selected. Informed consent was obtained from the patient for publication of this case report. She was hospitalized, due to aortic dissection found by ultrasonic examination in the pregnancy test. She did not have any history of hypertension or diabetes, but she had a medical history of "aortic dissection" in her family, specifically, her father died of aortic dissection. The patient didn't suffer from discomforts like chest distress or chest pain, tearing-type back pain, stomachache, nausea, or vomiting during the pregnancy.

\section{Auxiliary examination}

Ultrasonic examination of the patient in the pregnancy test indicated dissection from thoraco-abdominal aorta to two-side common iliac arteries (Figures 1, 2, 3, and 4). CTA indicated aortic dissection, the first cleavage was located at the T6-T7 level and stopped at two-side common iliac arteries,
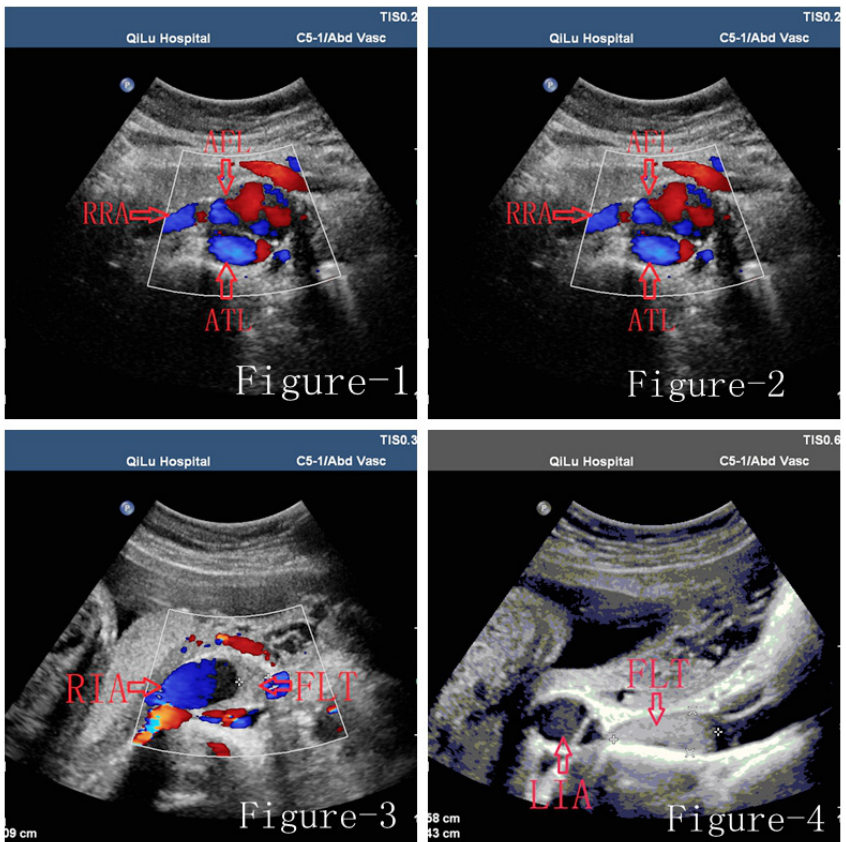

Figure 1-4. Ultrasonic examination of the patient in the pregnancy test indicated dissection from thoraco-abdominal aorta to two-side common iliac arteries. 

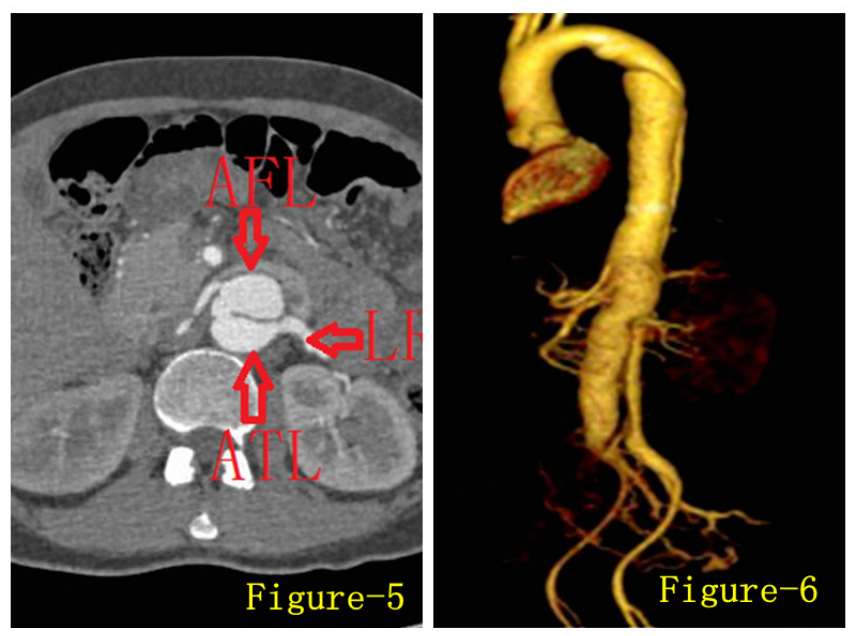

Figure 5-6. CTA indicated aortic dissection, the first cleavage was located at T6-T7 level and stopped at two-side common iliac arteries, and a linear low-density shadow was seen inside aortic lumen, presenting spiral descending trend. Celiac trunk, inferior mesenteric artery and right renal artery started from the false lumen, and superior mesenteric artery and left renal artery started from the true lumen.

and a linear low-density shadow was seen inside aortic lumen, presenting spiral descending trend. Celiac trunk, inferior mesenteric artery and right renal artery started from the false lumen, and superior mesenteric artery and left renal artery started from the true lumen (Figures 5 and 6).

Diagnosis was aortic dissection, Stanford B type, during 25+4-week pregnancy. Treatment involved endovascular repair of aortic dissection implementation under general anesthesia and hysterotomy delivery.

\section{RESULTS}

The patient accepted endovascular repair of aortic dissection (Figures 7 and 8) Following surgery, the patient and her family decided to terminate the pregnancy. She was then transferred to the maternity ward, and hysterotomy delivery was implemented. When her general postoperative state became good, the patient safely was discharged. A regular follow-up visit was paid to her, and the prognostic effect was favorable.

\section{DIsCussION}

\section{Etiology and patbogenesis}

Aortic dissection is a rarely seen complication in the gestation period. Pregnancy is one of dangerous factor for aortic dissection, which threatens the patient's life at any time. Up to half of all aortic dissections and ruptures in women younger than 40 years are associated with pregnancy [Smok 2014]. High estrogen/progesterone level, pregnancy-induced hypertension syndrome, hemodynamic change, and increasing circulating blood volume during the gestation period can
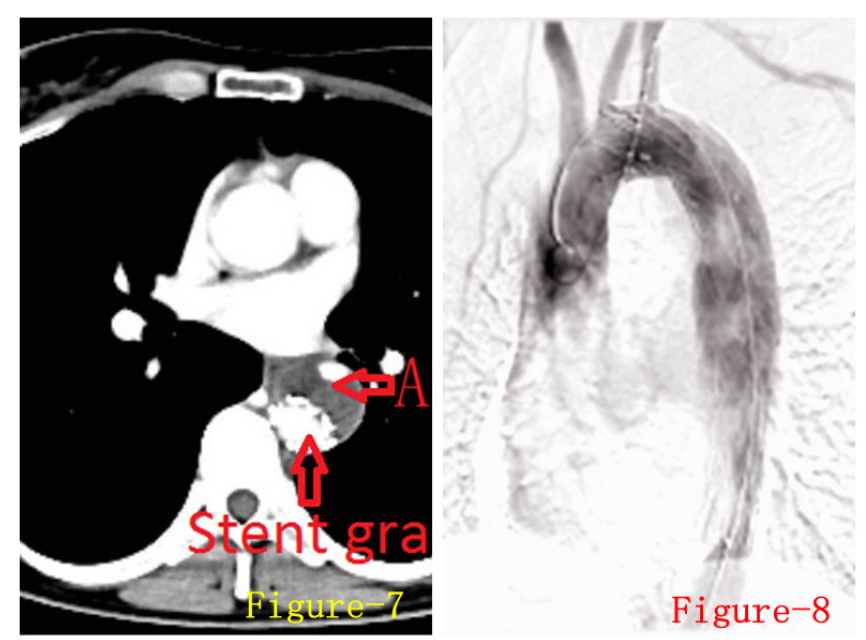

Figure 7-8. After accepting endovascular repair of aortic dissection.

aggravate susceptibility of the disease. Estrogen/progesterone accelerates synthesis and degradation of collagens and non-collagens at aortic walls so that the aorta is degraded and elastin is increased, which will affect tissue structure of the aortic wall; hemodynamic change enhances shear stress of blood flow toward blood vascular wall [Sanghavi 2014]. Genetic factors like Marfan syndrome, aortic middle-layer retrogression may be the main causes for aortic dissection, during the gestation period. Defects of connective tissues at the aortic wall result in middle-layer cystic deformation and reduce collagens and elastic fibers at the vascular wall, and consequently, the membrane lacks support and goes through rupture [Katsuragi 2011]. This patient was a young woman with a family history of the disease, namely her father died of aortic dissection. The patient did not have a hypertension or smoking history, and she was healthy before this. Occurrence of aortic dissection to this patient might be related to an abnormally developed artery or inheritance, not excluding genetic diseases like Marfan syndrome and congenital connective tissue diseases [Tefera 2010].

\section{Clinical manifestations and diagnosis}

Those accounting for $90 \%$ among the typical manifestations of aortic dissection are burst acute chest and back pain. Sixty-five percent to $75 \%$ patients are concurrent with intractable hypertension [Erbel 2014], including corresponding heart failure, myocardial infarction and cardiogenic shock, accompanied by corresponding clinical manifestations like hemoptysis, hematemesis, dyspnea, dizziness and headache, mental disorder, syncope, hemiplegic paralysis, and multiple organ failure, etc. Pregnancy concurrent with aortic dissection without typical clinical manifestations is rarely seen, and most often is considered chronic aortic dissection, so misdiagnosis easily will be made. Auxiliary examinations of aortic dissection include ultrasound, CT, MRI, etc. Ultrasonic test is affected by related factors like patient condition and examiner experience, so CTA or MRI examination should be selected, if conditions allow. 


\section{Treatment}

For pregnant patients concurrent with aortic dissection, a therapeutic schedule should be formulated, according to aorta typing, acute and chronic degree, general physical conditions of patients, gestational weeks, fetus state and will of the patient and their family. Therapeutic principles recommended by Zeebregts et al. [Zeebregts 1997] are as follows:

(1) Gestational weeks $<28$ weeks. Because it's difficult for the fetus to survive after delivery, operation is suggested and active miscarriage prevention should be implemented; however, if the patient and family members see that this disease is dangerous and give priority to the safety of the mother after deliberation, then hysterotomy delivery can be carried out.

(2) Gestational weeks 28-32 weeks. Whether the fetus will be delivered is decided according to acute and chronic degree of aortic dissection and fetus conditions. When clinical manifestations are serious and threaten life of the pregnant woman, caesarean section and aortic operation should be implemented immediately.

(3) Gestational weeks $>32$ weeks. Once aortic dissection is diagnosed, operative treatment of aorta should be implemented as soon as possible after stimulation of lung maturity and hysterotomy delivery. In addition, CTA examination is implemented as preoperative examination or endovascular interventional operation is carried out under an emergency.

Influences of X-ray and contrast medium on the fetus should be considered [Shu 2014]. Woussen et al. [Woussen 2016] believe that radiation dosage in the early pregnancy that is greater than $150 \mathrm{mGy}$ will result in teratogenesis of the embryo or fetus. The dosage in CTA examination before definite diagnosis is far lower than teratogenesis dosage, but it can't be more prudent in the early pregnancy. For this pregnant patient concurrent with Stanford B type aortic dissection, she accepted "endovascular repair operation using covered stents." Because this patient and her family members decided to terminate the pregnancy through deliberation, hysterotomy delivery was implemented. Meanwhile, further thinking was needed during the treatment of similar patients. This patient had no clinical manifestation, antenatal examination found that thrombosis happened in false lumen, and she was considered with chronic $\mathrm{AD}$ with relatively stable state of the illness. Close observation was needed to decide whether drugs would be taken for effective control. After gestational weeks exceeded 32 weeks, caesarean section and endovascular repair operation were simultaneously implemented. Of course, as gestational weeks increased, this treatment would be of extreme risk with increasing circulating blood volume, hemodynamic change, and all kinds of unpredictability. The success depended on determination and courage of the doctor and patient.

\section{CONCLUSION}

Not all patients with aortic dissection have typical clinical symptoms. This disease should correctly be understood to prevent missed diagnosis and misdiagnosis and timely to save the patient's life. Aortic dissection is an extremely dangerous complication during the gestation period. Timely diagnosis of this patient benefited from carefulness and superb technology of the sonographer in this hospital. Meanwhile, obstetrician recognition of aortic dissection should be enhanced. When obstetrician encounters a patient suffering from chest and back pain or hypertension, this disease should be taken into consideration even if the occurrence rate of aortic dissection is extremely low. Meanwhile, the doctor should be vigilant with asymptomatic aortic dissection, inquire about medical history, previous history and family history, and carefully conduct a physical examination. Moreover, the doctor should carry out ultrasonic cardiogram examination for timely diagnosis and early treatment, when necessary. Multidisciplinary joint decisions should be made so as to save lives of the patient and her fetus.

\section{ACKNOWLEDGEMENT}

The authors would like to express their gratitude to Dr. Jianjun Jiang for the assistance given in the endovascular repair operation.

\section{REFERENCES}

Braverman AC. 2010. Acute aortic dissection: clinician update[J]. Circulation. 122(2):184-188.

Erbel R, Aboyans V, Boileau C, et al. 2014. 2014 ESC guidelines on the diagnosis and treatment of aortic diseases [J]. Eur Heart J. 35 (41) :2873-2926.

Katsuragi S, Ueda K, Yamanaka K, et al. 2011. Pregnancy-associated aortic dilatation or dissection in Japanese women with Marfan syndrome [J]. Circ. 75(11):2545-2551.

Katz NM, Collea JV, Moront MG, et al. 1984. Aortic dissection during pregnancy: treatment by emergency cesarean section immediately followed by operative repair of the aortic dissection [J]. Am J Cardiol. 54(6):699-701.

Sanghavi M, Rutherford JD. 2014. Cardiovascular physiology of pregnancy [J]. Circulation. 130 (12): 1003-1008.

Shu C, Fang K, Dardik A, et al. 2014. Pregnancy associated type B aortic dissection treated with thoracic endovascular aneurysm repair [J]. Ann Thorac Surg. 97 (2): 582-587.

Smok DA. 2014. Aortopathy in pregnancy [J]. Semin Perinatol. 38(5):295-303.

Tefera G. 2010. Traumatic thoracic aortic injury and ruptures [J]. J Vasc Surg. 52:41S-44s.

Woussen S, Lopez-Rendon X, Vanbeckevoort D, et al. 2016. Clinical indications and radiation doses to the conceptus associated with CT imaging in pregnancy: a retrospective study [J]. Eur Radiol. 26(4):979-985.

Zeebregts CJ, Schepens MA, Hameeteman TM, et al. 1997. Acute aortic dissection complicating pregnancy [J]. Ann Thorac Surg. 64(5):1345-1348. 